\title{
Redes sociales y educación
}

\section{Social networks and education}

\section{Editorial invitado}

Maria Rosa Buxarrais

Departamento de Teoría e Historia de la Educación. Universidad de Barcelona, España.mrbuxarrais@ub.edu

La relación entre las TIC y la Educación ha sido ya ampliamente abordada desde varios puntos de vista por parte de la academia. En general, el argumento que ha motivado y orientado esos estudios expresa que la irrupción de las TIC en el escenario educativo está reformulando lo que hasta ahora entendíamos por educación, e incluso nos ha hecho reflexionar seriamente sobre la viabilidad de las instituciones educativas tradicionales (Buxarrais \& Ovide, 2011).

Este inédito cambio en la historia tecnológica de la humanidad trasciende el ámbito educativo y, actualmente, tiene consecuencias incluso para el desarrollo económico global, el bienestar público y social, la convivencia pacífica intercultural y la participación cívica y política (G8 Heads of State, 2000). Varios organismos internacionales han declarado que las TIC son una herramienta estratégica para que las sociedades contemporáneas logren las oportunidades de crecimiento y garantizar, así, una oportuna y adecuada participación de las y los estudiantes en el mercado laboral actual y en la vida política y social contemporánea (Organization for Economic Co-operation and Development [OECD], 2001; Organization of Economic Co-operation and Development [OECD], 2006; United Nations, 2005; World Bank, 2003).

En efecto, las sociedades postindustrializadas están realizando varias reformulaciones educativas con tal de aprovechar las bondades y ventajas que estos flamantes recursos, y los particulares ambientes de aprendizaje que ofrecen. Estas reformulaciones están basadas, principalmente, en la ruptura que existe entre la educación tradicional, originada y vinculada a la producción industrial, y el modelo educativo propio de las sociedades contemporáneas, llamadas Sociedades del Conocimiento, y que se podría esquematizar de la siguiente manera:

\begin{tabular}{|c|c|}
\hline Educación adaptada a la producción en masa & $\begin{array}{c}\text { Educación adaptada a la creación del } \\
\text { conocimiento }\end{array}$ \\
\hline $\begin{array}{c}\text { Un número reducido de personas adquiere } \\
\text { capacidades avanzadas y un gran número } \\
\text { adquiere capacidades mínimas }\end{array}$ & $\begin{array}{c}\text { Un gran número de personas adquieren } \\
\text { capacidades avanzadas }\end{array}$ \\
\hline $\begin{array}{c}\text { Estandarización de los procesos y de los } \\
\text { resultados }\end{array}$ & $\begin{array}{c}\text { Personalización de los procesos y diversidad de } \\
\text { resultados }\end{array}$ \\
\hline
\end{tabular}




\begin{tabular}{|c|c|}
\hline Educación adaptada a la producción en masa & $\begin{array}{c}\text { Educación adaptada a la creación del } \\
\text { conocimiento }\end{array}$ \\
\hline $\begin{array}{c}\text { El conocimiento disciplinar es el centro } \\
\text { curricular }\end{array}$ & $\begin{array}{c}\text { El conocimiento disciplinar crece por otras } \\
\text { capacidades, como la colaboración, la } \\
\text { comunicación y la resolución de problemas }\end{array}$ \\
\hline Conocimiento como hecho establecido & El conocimiento se ha de crear \\
\hline $\begin{array}{c}\text { El profesor como autoridad central y } \\
\text { diseminador de información }\end{array}$ & $\begin{array}{c}\text { Los alumnos como estudiantes intencionales y } \\
\text { creadores de conocimiento }\end{array}$ \\
\hline Lugar de trabajo individual & Proyectos colaborativos \\
\hline Tecnología como suplemento & Tecnología como eje central \\
\hline $\begin{array}{c}\text { Centralización de la educación y escuelas } \\
\text { estructuradas de manera jerárquica }\end{array}$ & $\begin{array}{c}\text { Descentralización de la educación y escuelas } \\
\text { estructuradas de manera horizontal; más } \\
\text { autonomía del profesorado }\end{array}$ \\
\hline
\end{tabular}

Fuente: (Kozma, 2011)

Aunque existe un amplio consenso respecto a reformular la educación debido a la influencia y las prometedoras posibilidades que ofrecen las TIC, pero específicamente en el uso de las redes sociales. Todavía no hay suficientes estudios que confirmen sus bondades. De ahí que hayamos pensado en un monográfico que nos ilumine y oriente.

Es evidente que el uso de las redes sociales por parte de los jóvenes -y no tan jóvenes - aumenta día tras día de forma acelerada. Cada vez son más los usuarios de dichas redes en su vida cotidiana. Parece que ya no podemos concebir nuestra vida sin ellas. Muchos estaríamos de acuerdo en afirmar que vivimos en la "era de la hiperconectividad" (Reig y Vilchez, 2013).

Sin lugar a dudas, las redes sociales son herramientas clave para la comunicación en muchos ámbitos, incluyendo el educativo. Además, somos conscientes de su potencial, no solamente porque promueven nuevas formas de aprendizaje, como el aprendizaje colaborativo, sino porque nos ofrecen todo un mundo de información y posibilidades de interconectividad que pueden ser positivas o negativas, según se mire. Por lo tanto, la influencia de las redes sociales en la educación es sumamente importante, por lo que es necesario que las personas aprendamos a integrarlas.

Las redes sociales, entendidas como sistemas que permiten establecer relaciones con otros usuarios (Prato, 2010), han sido objeto de estudio desde diferentes enfoques, principalmente desde una perspectiva sociológica. En el terreno de la educación ha surgido un interés reciente por conocer e incorporarlas en los ámbitos académicos, aportando evidencias científicas que analizan su uso y las consecuencias hasta este momento. De todas formas, son necesarias más investigaciones que planteen los puntos fuertes y débiles de su uso. El incremento significativo de su uso en los últimos años nos obliga, como teóricos de la educación, a detenernos en su análisis.

Este monográfico reúne artículos relacionados con la temática que nos ocupa, que se centran en 
diversos aspectos, sin duda complementarios, tanto a nivel de ámbito de aplicación como de enfoque práctico que se deriva: desde el uso de las redes sociales como herramienta para la investigación universitaria en la historia de la educación, al diseño de un nuevo modelo de universidad con un profesorado acorde a las circunstancias, así como unos estudiantes que utilizan dichas redes para construir conocimientos y generar en ellos una actitud de colaboración y crítica, para terminar con una visión acorde con los tiempos que corren: la necesidad de desconectar por cuestiones de salud mental y espiritual.

La posibilidad de gestión de la información tiene un poder relevante en las redes sociales. Esto significa que puede ponerse al alcance de muchos investigadores que quieren fortalecer el conocimiento y el aprendizaje. Así pues, el primer artículo contenido en este monográfico (Payà, Duart y Mengual, 2016) sugiere el uso de las redes sociales para la investigación universitaria de la historia de la educación, una historia de la educación 2.0 propia de la sociedad digital. Sabemos que las redes sociales generan múltiples oportunidades para aprender y construir el conocimiento de manera compartida en los distintos ámbitos de conocimiento. Aquí se centran en el conocimiento histórico-educativo.

A pesar del aumento del número de usuarios de las redes sociales, será necesario superar muchas limitaciones para su uso eficaz en el ámbito académico por parte de profesoresy alumnos. Seguramente, las funciones de los docentes tendrán que repensarse y modificarse si se plantea el uso de las redes sociales en el ámbito académico: ¿cómo se transmitirá el conocimiento?, ¿seguirán haciendo falta docentes con habilidades comunicativas o capacidad para la retórica?, ¿se enfatizará la autonomía del estudiantes en sus aprendizajes?, ¿cómo se concebirá un docente en esta nueva universidad?, ¿hasta qué punto pueden y podrán utilizarse las redes sociales para interaccionar o comunicarse con los estudiantes?, estas y otras cuestiones habrá que planteárselas y ameritan un debate entre los docentes universitarios para que se tomen decisiones consensuadas y con sentido.

En el artículo de Fuentes, Esteban y González (2016) se nos confirma que estamos asistiendo a una transformación de la universidad de la que no podemos hacer caso omiso: una universidad que debe afrontar su responsabilidad social de manera más intensa, que se convierte en un espacio de conocimiento e investigación global, con unos docentes conocedores y practicantes del uso educativo de las TIC y las redes sociales. En esta universidad no se tratará de acumular contenidos sin más, sino que los estudiantes tendrán que hacerse preguntas, relacionar contenidos con aprendizajes previos, desarrollando un pensamiento autónomo y crítico para elaborar un criterio propio teniendo en cuenta las cuestiones éticas.

La universidad se enfrenta a aulas de nativos digitales que demandan un nuevo tipo de enseñanza (Gómez-Aguilar, Roses-Campos y Farias-Batlle, 2012). Por esto, deberá cuestionarse, entre otras cosas, el sistema de organización de las materias en determinado uso horario o calendario. El 
aprendizaje no se da solamente en las aulas, en unas sesiones de clase, sino que puede adquirirse en otros dispositivos. "En las redes sociales educativas los usuarios comparten sus conocimientos sobre una determinada materia o disciplina, muestran sus trabajos y ponen su experiencia a disposición de los demás, ayudándoles en tareas específicas y a través de atención personalizada" (Abuin, 2009, p. 2). Incluso, profesores y estudiantes modifican su forma de comunicarse e interaccionar, en algunos momentos no existe el cara a cara.

Uno de los grandes inconvenientes de las redes sociales radica en que las universidades no asumen el cambio que se está produciendo en la red. Ni profesores ni estudiantes contraen dicha responsabilidad en el cambio de la dinámica educativa. En este sentido, podemos advertir las resistencias de los estudiantes en el estudio de González, Espuny, Leixà i Gisbert (2016) sobre las actitudes de los estudiantes universitarios hacia el uso educativo de las redes sociales, cuyos resultados corroboran el hecho de que pasan mucho tiempo usando las redes sociales en la vida personal, pero poco en la académica, debido a la falta de propuestas por parte de los docentes. Así pues, el uso de las redes sociales para actividades académicas casi siempre parte de la iniciativa de los estudiantes y no del profesor.

Por otra parte, el desfase generacional entre estudiantes (nativos digitales) y profesores (inmigrantes digitales) hace necesario que el profesor sea experto no únicamente en los contenidos que pretende transmitir en su materia sino que, además, reciba formación y desarrolle destrezas en el uso y manejo de estas herramientas, adaptándose a nuevos entornos (Duart, 2009). En este sentido, los roles de los docentes están cambiando del control a la influencia sutil y/o conformación inicial (Siemens, 2010). Eso significa que el profesor, a parte de ser consciente de la necesidad de realizar cambios metodológicos y no aferrarse a procedimientos y estrategias del pasado, debe saber cómo formar el criterio de sus estudiantes, cómo mediar entre los contenidos y el aprendizaje de competencias y cómo adquirir y aplicar competencias digitales centradas en su disciplina.

Nuestra experiencia en este ámbito ${ }^{1}$ nos confirma que el uso de las redes sociales en las aulas posee una serie de ventajas que deben hacernos plantear su introducción en asignaturas de las ciencias humanas y sociales, porque:

1) Permiten la transmisión de conocimientos y la colaboración entre personas, además de desarrollar las competencias digitales imprescindibles hoy para cualquier profesional de la educación.

2) Posibilitan el desarrollo de habilidades y aptitudes para el trabajo en equipo y la importancia de la colaboración.

\footnotetext{
1 En la asignatura Axiologia y educación en valores, obligatoria de $3^{\circ}$ del Grado de Pedagogia, hemos introducido el uso de las redes sociales: Facebook y blog, y los resultados confirman lo que se ha concluido en otros estudios sobre actitudes de los estudiantes hacia las redes sociales.
} 
3) Motivan a los estudiantes a la hora de tratar temáticas relacionadas con la asignatura, porque promueven su participación activa y les proporciona oportunidades para desarrollar competencias éticas.

4) Promueven su autonomía en cuanto a la búsqueda y selección de material complementario para la asignatura.

5) Se convierten en herramientas interactivas y eficaces para la enseñanza-aprendizaje (foros, blogs, Facebook, Twiter, YouTube, etc.), fomentando el diálogo y la participación.

Pero cuando el uso de las redes sociales se lleva al límite y nuestras vidas, especialmente las de los jóvenes, no pueden entenderse sin la conectividad permanente o hiperconectividad, debemos empezar a considerar la necesidad y la repercusión de la tecnología en ellas. Porque, sin duda, se crea un hábito difícil de romper, incluso puede derivar en una adicción o el "síndrome FOMO" como nos relatan Naval, Serrano-Puche y Sádaba (2016). Plantean la instauración de momentos de desconexión digital para promocionar y cultivar la comunicación cara a cara. Desde un punto de vista educativo, se ha planteado la necesidad de enseñar competencias digitales y mediáticas. Pero a parte de educar para el uso de las TIC, fomentando la capacidad de discernir y valorar críticamente la información que proviene de las redes sociales, será necesario incidir en un fenómeno sobre el que hoy en día se está debatiendo: el unplugging, un movimiento que se relaciona con otro movimiento (el movimiento slow). Se presenta otro debate actual sobre el tema: el multitasking, del cual se dice que merma la capacidad de pensar de manera profunda y creativa.

En conclusión, podremos constatar con la lectura de los trabajos recogidos en este monográfico que el uso de redes sociales en el ámbito académico puede generar cambios imprevistos en las formas de enseñar y aprender e investigar, que no serán fácilmente resueltos, pero seguramente van a enriquecer y modificar nuestra forma de afrontar la investigación y la docencia. Si no aspiramos a modificar nuestra práctica docente y nuestra forma de investigar, mejor olvidarnos de incorporar a las redes sociales.

\section{Referencias}

Abuin, N. (2009). Las redes sociales como herramienta educativa en el ámbito universitario. Disponible en: http://moodle.upm.es/adamadrid/file.php/1/web_IV_jornadas_ADA/comunicaciones/30_Abuin. pdf [15 de enero del 2016].

Buxarrais, M. R., \& Ovide, E. (2011) El impacto de las nuevas tecnologías en la educación en valores del siglo XXI. En Sinéctica, http://www.scielo.org.mx/pdf/sine/n37/n37a2.pdf 
Duart, J.M. (2009). Internet, redes sociales y educación. Revista Universidad y Sociedad del Conocimiento, 6, 1; 1-2.

Fernández, I. (2012). Potencialidad educativa de las redes sociales. Revista Iberoamericana para la Investigación y el Desarrollo Educativo, 8(1). Disponible en: <http://www.ride.org.mx/pdf/ globalizacion/02_globalizacion.pdf > [15 de enero del 2016].

Gómez, M., Roses, S., \& Farías, P. (2012). El uso académico de las redes sociales en universitarios. Revista Científica de Comunicación y Educación, 19(38):131-138.

Kozma, R. (2011). A framework for ICT policies to transform education. In R. Kozma (Ed), Transforming education: The power of ICT policies (pp. 27-24). Paris: UNESCO.

Prato, L.; Villoria, L. (2010. Web 2.0: Redes Sociales. Argentina: Editorial Universidad Nacional de Villa María.

Sánchez Rodríguez, J., Rioz-Palmero, J., \& Sánchez-Rivas, E. (2015). Uso problemático de las redes sociales en estudiantes universitarios. Revista Complutense de Educación, 26, 159-174. doi: http:// dx.doi.org/10.5209/rev_rced.2015.v26.46360

Siemens, G. (2010). My personal learning network is the most awesome thing ever. Elearn space. Blog. http://www.elearnspace.org/blog/2010/09/30/web-3-0xweb/

Spector, J. M., Merrill, M. D., Elen, J., \& Bishop, M. J. (Eds) (2013). Handbook of Research on Educational Communications and Technology. New York: Springer. 